RUNNING HEAD: IMPLICIT EVALUATION

\title{
A Propositional Model of Implicit Evaluation
}

\author{
Jan De Houwer
}

Ghent University, Belgium

In press. Social Psychology and Personality Compass.

mailing address:

\author{
Jan De Houwer \\ Department of Experimental Clinical and Health Psychology \\ Ghent University \\ Henri Dunantlaan 2 \\ B-9000 Ghent \\ Belgium \\ Email: Jan.DeHouwer@UGent.be \\ Tel: ++329 2646462 \\ Fax: ++3292646489
}

Acknowledgements

Jan De Houwer, Ghent University, Belgium. Preparation of this paper was supported by Ghent University Methusalem Grant BOF09/01M00209 and by the Interuniversity Attraction Poles Program initiated by the Belgian Science Policy Office (IUAPVII/33). The ideas put forward in this paper were also presented at the 4th EASP Meeting on the Psychology of Attitudes (Ghent, Belgium, May 2012) and the 2012 PMIG meeting (New Braunfels, USA, October 2012). Thanks to Adriaan Spruyt for comments and to Frans De Bie and Yvonne Vansweevelt for facilitating the writing process. 


\begin{abstract}
Implicit evaluation can be defined as the automatic effect of stimuli on evaluative responses. A major advantage of this definition is that it is neutral with regard to the mental processes and representations that mediate implicit evaluation. Whereas many existing models postulate that implicit evaluation is mediated by the automatic spreading of activation along associations in memory, it is also possible to entertain the idea that implicit evaluation is due to the automatic formation or activation of propositions. In line with such a propositional model of implicit evaluation, evidence suggests that implicit evaluation (a) can be based on instructions and inferences, (b) is sensitive to information about how stimuli are related, and (c) can reflect several propositions that differ only with regard to how stimuli are related. Although it might be difficult to differentiate between propositional models on the one hand and association-activation or dual-process models on the other hand, merely considering the idea that implicit evaluation might be mediated by propositions offers a new perspective on existing findings and leads to novel predictions about the conditions under which implicit evaluation occurs.
\end{abstract}

Keywords: evaluation, attitudes, automaticity, implicit processing, implicit attitudes 


\section{A Propositional Model of Implicit Evaluation}

One of the most basic principles of behavior is that living organisms respond in an evaluative manner to stimuli in the environment. For instance, stimuli that promote survival tend to evoke approach responses whereas stimuli that endanger survival tend to induce avoidance responses. The term "evaluation” has been used refer to this impact of stimuli in the environment on evaluative responses (De Houwer, 2009a; De Houwer, Gawronski, Barnes-Holmes, in press; Eagly \& Chaiken, 2007, p. 583). By studying the variables that moderate evaluation (i.e., that determine whether stimuli have an impact on evaluative responses as well as the nature of this impact), cognitive psychologists try to uncover the mental processes that mediate evaluation (i.e., the activation and transformation of mental representations via which stimuli in the environment influence evaluative responses).

In this paper, I focus on instances of implicit evaluation. The term "implicit evaluation" has been used to refer to a subset of all instances of evaluation, namely those in which stimuli have an automatic impact on evaluative responses (e.g. De Houwer, 2009a; De Houwer et al., in press; Gawronski \& Bodenhausen, 2011). More specifically, implicit evaluation is said to occur when stimuli influence evaluative responses under suboptimal conditions (e.g., even when there is little time, when the organism is engaged in other tasks, or when the person cannot discriminate between the presence or absence of the stimuli). A number of paradigms have been used to study implicit evaluation. For instance, in a typical evaluative priming task, a prime and a target are presented on each trial. Participants are asked to categorize the target words as good or bad while ignoring the primes. Most often, responses are faster when a target is preceded by a prime with the same valence (e.g., HAPPY - FLOWER) compared to a prime with a different valence (e.g., HAPPY CANCER; see Herring et al., 2013, for a review). In this paradigm, the evaluative 
categorization of a positive or negative target can be conceived of as an evaluative response. The impact of the primes on the speed with which these evaluative responses are emitted, is thus an instance of evaluation that can be qualified as implicit because it occurs despite the brief time between the onset of the prime and the onset of the target and despite the instruction to ignore the primes.

As I discussed elsewhere, defining (implicit) evaluation as the (automatic) effect of stimuli on evaluative responses has several important advantages (De Houwer, 2009a; De Houwer et al., in press). One major advantage is that such a definition does not commit itself to any ideas about the specific mental processes and representations by which stimuli in the environment (automatically) influence evaluative responses. Of course, there must be some kind of mental mechanism that mediates evaluation. However, the definition of evaluation is compatible with all possible theories about the mental processes that mediate evaluation. The high level of theoretical freedom that is offered by the definition of implicit evaluation stands in marked contrast with the dominance of association-activation models of implicit evaluation. In the first section of this paper, I describe some of the core assumptions of association-activation models. Afterwards, I discuss an alternative, propositional model according to which implicit evaluation is mediated by the automatic formation or activation of propositions. I also point out that entertaining propositional models has merit because it provides a novel perspective on known findings (i.e., heuristic value) and generates new predictions about the conditions under which implicit evaluation occurs (i.e., predictive value). In the second section, I summarize three lines of research that provided support for the novel predictions of propositional models of implicit evaluation.

\section{Cognitive Models of Implicit Evaluation}

\section{Association-Activation Models}


Association-activation models originate from semantic network models according to which long-term memory consists of interconnected nodes, each of which represents a certain concept (e.g., Collins \& Loftus, 1975). Importantly, when a node is activated, some of the activation spreads automatically to other nodes that are associated with the first node. The stronger the association, the more activation will spread between the associated concepts. Typically, it is assumed that associations in memory are formed gradually as the result of many experiences.

Assuming that positive valence and negative valence are represented as nodes in the network, one can conceptualize an attitude as an evaluative association, that is, as an association between the target concept and the nodes representing positive and negative valence (e.g., Fazio, 2001, 2007). In its most basic form, implicit evaluation can then be explained in the following manner: A stimulus in the environment activates the corresponding node in semantic memory, which in its turn automatically activates the node representing positive or negative valence, which in its turn influences a certain evaluative response. Consider a specific example of evaluation in which the sight of an ice cream cone (i.e., a stimulus) automatically evokes a smile (i.e., an evaluative response; see Figure 1A). Association-activation models specify one possible mental mechanism by which the ice cream cone could produce a smile: the ice cream cone that is present in the environment somehow activates the representation of ice cream in memory, which automatically activates the concept "good" via the strong association between the nodes representing ice cream and positive valence, which in its turn produces a positive facial expression (see Figure 1B). Likewise, evaluative priming effects would arise because activation of the node representing the prime (e.g., HAPPY) automatically activates the node of positive valence which either facilitates the selection of positive responses (relative to negative responses) or lowers the 
threshold for the activation of nodes representing other positive target concepts (e.g., FLOWER) relative to nodes representing negative target concepts (e.g., CANCER; see Herring et al., 2013, for a review).

This simple but elegant model explains not only the fact that evaluation can occur automatically (i.e., by virtue of the process of automatic spreading of activation) but also leads to the prediction that implicit evaluation should be moderated by variables that influence the strength of evaluative associations, a prediction that has received empirical support (e.g., Fazio, Sanbonmatsu, Powell, \& Kardes, 1986).

\section{Propositional Models}

Propositions are statements about the world. For instance, the proposition "I am good" is a statement about the properties of oneself. Importantly, as statements about world, propositions specify the nature of the relation between concepts. For instance, the propositions "I am good" and "I want to be good" both involve the concepts "I" and "good" but differ in the way in which these concepts are said to be related. Hence, a vital difference between propositions and associations is that propositions contain relational information, that is, information about how concepts are related. It is often also assumed that propositions about events can be formed not only on the basis of the repeated experience of those events but also as the result of a single instruction or inference concerning those events (see De Houwer, 2009b; De Houwer, in press; Lagnado, Waldmann, Hagmayer, \& Sloman, 2007; Mitchell, De Houwer, \& Lovibond, 2009).

Propositional processes are typically thought to operate in a non-automatic manner (e.g., Strack \& Deutsch, 2004) and are therefore hardly ever considered to be plausible mediators of automatic effects. Nevertheless, the idea that propositions can be formed or activated automatically is not unreasonable. First, there is evidence showing that propositions 
can be formed automatically as the result of automatic task misapplication. For instance, in a recent series of studies, my colleagues and I presented on each trial two line drawings that were similar in size but that depicted objects of different size (e.g., a bike and an airplane). Participants were asked to judge the size of the second object (i.e., the target) relative to the size of a particular reference object (e.g., to determine whether an airplane is bigger or smaller than a football). The size of the reference object could vary from block to block or from trial to trial (e.g., on some trials it was a football, on others it was a car). The first line drawing (i.e., the prime) that preceded the target depicted an object that was smaller or bigger than the reference object. Responses were faster when prime and target had the same compared to a different size relative to the reference object. Importantly, the same prime facilitated "bigger than" responses when it was bigger than the reference object on that trial (e.g., when the prime was a bike and the reference object a football) and "smaller than" responses when it was smaller than reference object on that trial (e.g., when the prime was a bike and the reference object a car). This result suggests that participants applied the target task also to the primes, which resulted in the formation of propositions about the size of the primes (e.g., "the bike is bigger than the football”). Because participants were told to respond only to the targets and because the effects arose even though the primes were presented only briefly, we concluded that the application of the target task to the primes was automatic in the sense of unintentional and fast (Heider, Spruyt, \& De Houwer, 2014; see Dehaene et al., 1998, and Van Opstal, Gevers, Osman, \& Verguts, 2010, for findings showing that task misapplication does not even require awareness of the prime stimuli). Within the context of the present paper, these findings are important because they reveal a mechanism (i.e., task misapplication) via which propositions can be formed automatically. This lends credence to the claim that (the automatic formation of) propositions can mediate implicit evaluation. 
Second, propositions cannot only be formed automatically via processes such as task misapplication; they can also be retrieved automatically from memory. For instance, one could assume that propositions are encoded in memory as episodic-like representations that (unlike associations) encode relational information (see Hintzman, 1986, for one possible model). Like all episodic representations, such episodes can be retrieved from memory automatically. Therefore, propositions (e.g., “ice cream is good”) might be retrieved even when they are unwanted and no longer considered to be valid (e.g., when dieting).

A propositional model of implicit evaluation postulates that a stimulus can evoke an evaluative response automatically only after a proposition about the evaluative properties of the stimulus has been formed or activated automatically (Hughes, Barnes-Holmes, \& De Houwer, 2011). As I noted in the previous paragraphs, there might be different processes by which propositions can be formed or activated automatically. For instance, the sight of ice cream on a hot summer day could automatically retrieve memories in which the proposition “ice cream is good" was encoded (see Figure 1C). Instances of implicit evaluation such as evaluative priming effects, on the other hand, might be based on a process of task misapplication, in which the task of evaluating the target is misapplied to the prime. Such a task misapplication would facilitate responses when prime and target have the same valence but delay responding when they differ in valence. In fact, as noted by Klauer, Musch, and Eder (2005, p. 902), a task misapplication account of evaluative priming effects is in line with many findings in the evaluative priming literature (see Klauer, Teige-Mocigemba, \& Spruyt, 2009, for a detailed model of evaluative priming that could be interpreted as an instantiation of the principle of task misapplication).

\section{What Is the Added Value of Propositional Models?}

The propositional model of implicit evaluation that is championed in this paper (also 
see Hughes et al., 2011) is meant to challenge association-activation models of implicit evaluation. However, in order to avoid misguided debates, it is important to be explicit about what exactly is being challenged. As stated earlier on in the paper, propositions are statements about the world that contain information about the way in which concepts are related (e.g., "I am good” vs. "I want to be good”). Some might argue that it is possible to devise a semantic network of associations that operates on the principle of spreading of activation but that does encode information about the way in which concepts are related. For instance, one might allow for nodes that encode relational information (e.g., one node encoding the goal "I want to be good" and another node encoding the belief "I am good”). Likewise, perhaps models that incorporate a distributed associative memory network (e.g., Gawronski \& Bodenhausen, 2011) can be modified in such a way that the memory network encodes relational information. I would consider such models to be specific instantiations of propositional models rather than as rivals of propositional models. In my opinion, propositional models challenge the idea that implicit evaluation is mediated by simple, unqualified associations between conceptual nodes or any other mental representations that are unable to represent relational information. As such, one of the main contributions of propositional models is the proposal that relational information is potentially important for understanding implicit evaluation. The fact that simple association-activation models have until now dominated thinking about implicit evaluation and that, as a consequence, the role of relational information has so far received little attention in research on implicit evaluation is perhaps surprising, especially because the role of relational information has been studied in related research areas such as research on goal-directed behavior (e.g., Aarts \& Elliot, 2012) and the representation of the self (e.g., Markus, 1977). By putting forward the idea that implicit evaluation could be mediated by the automatic formation or retrieval of propositions, 
the present paper hopes to highlight the potential importance of relational information also for research on implicit evaluation.

More generally, propositional models have merit because they provide a new perspective on old findings (i.e., they have heuristic value) and generate new predictions about the variables that moderate implicit evaluation (i.e., they have predictive value). In the next section, I discuss a number of findings that were predicted on the basis of the idea that propositions mediate implicit evaluation. It is likely that some of these findings can be explained in a post-hoc manner by some variants of association-activation models or variants of dual-process models that allow for both associations and propositions (e.g., Rydell \& McConnell, 2006). In fact, as argued by Miller and Escobar (2001; also see Garcia-Marques \& Ferreira, 2011), it is always difficult to distinguish between broad classes of models that each can be instantiated in different ways. As a class of models, not only associationactivation and dual-process models but also propositional models are too unconstrained to allow for a straightforward falsification. For instance, the idea that propositions can be activated automatically from memory allows propositional models to mimic the behavior of an association-based network. Assume that a stimulus in the environment (e.g., a particular medicine) automatically activates a proposition in memory (e.g., the proposition that the medicine cures cancer). Under certain conditions, only the related concepts of the proposition might be retrieved (e.g., “medicine” and “cancer”) but not the relational information (e.g., “cures”). Under those conditions, implicit evaluation would seem to be unaffected by relational information (e.g., the medicine might evoke a negative rather than a positive implicit evaluation) even though the evaluation is mediated by the (partial) activation of a propositional representation. Although this idea generates a number of interesting testable hypotheses (e.g., that the impact of relational information depends on the extent to which the 
environment acts as a retrieval cue for that information), it would be difficult to disentangle a propositional model that allows for such a partial automatic retrieval from a dual-process model that allows for both propositions and associations as mediators of implicit evaluation (see Gawronski \& Bodenhausen, in press, for an excellent discussion of the pragmatic limits in the falsification of theories in general).

Nevertheless, even if propositional (or association-activation or dual-process) models can never be proven true or be falsified, it remains interesting to exploit the heuristic and predictive power of these models. As illustrated by the studies that are described in the next section of the paper, merely entertaining the possibility that propositions mediate implicit evaluation provides a very different perspective on implicit evaluation. After decades of dominance by association-activation models, this novel perspective can provide a new impetus for research on implicit evaluation.

\section{Evidence for Propositional Models of Implicit Evaluation}

Propositions have two interesting properties: (1) they can be formed as the result of a single instruction or inference and (2) they contain relational information. Hence, if propositions mediate implicit evaluation, then implicit evaluation (1) should occur even for stimuli whose evaluative properties have been learned via a single instruction or inference, (2) should depend on relational information, and (3) might reflect different types of propositions that differ only with regard to relational information. In this section, I provide a brief review of studies in which these predictions of propositional models were tested.

\section{Implicit Evaluation on the Basis of Individual Instructions and Inferences}

Instruction-based implicit evaluation has been examined by giving participants a single instruction about the properties of novel stimuli and then testing whether these stimuli automatically influence evaluative responses. Because the stimulus is novel, any impact of 
the stimulus on evaluative responses is likely to be mediated by the proposition that was created on the basis of the instruction. In one study (De Houwer, 2006; see Gast \& De Houwer, 2013, Gregg, Seibt, \& Banaji, 2006, and Smith, De Houwer, \& Nosek, 2013, for related results), participants were told that they would see a series of pleasant and unpleasant pictures, each of which would be preceded by a meaningless word. The instructions then revealed the two words that would always precede positive pictures and the two words that would always precede negative pictures. However, before the words and pictures were actually presented, participants completed an Implicit Association Test (IAT) that was designed to capture the implicit evaluation of the meaningless words. Results showed that a single instruction about the relation between the meaningless words and positive or negative pictures was sufficient to influence the implicit evaluation of the words: The words that were said to be related to positive pictures were liked more than those that were said to be followed by negative pictures.

Gast and De Houwer (2012) went on to examine inference-based implicit evaluation. Rather than giving instructions about novel stimuli, participants received information from which they could infer propositions about the properties of novel stimuli. In a first study, positive pictures were paired with a grey square on which the number 1 was printed whereas negative pictures always co-occurred with a grey square on which the number 2 was printed. Afterwards, participants were shown two neutral pictures and were told that during the previous presentations of the pictures and squares, grey squares with the number 1 always covered one of those neutral picture whereas grey squares with the number 2 always covered the other neutral picture. A subsequent IAT revealed that participant preferred the first neutral picture over the second one even though the neutral pictures had never actually been paired with the positive and negative pictures. In a second study, participants first ate pieces of 
cookie from two bowls while being unable to feel or see the shape of the cookies. The pieces of cookie from one bowl tasted bad whereas the other cookies tasted good. During a second phase, participants no longer tasted the cookies but were allowed to see the shape of the cookies. By combining the information from both phases, they could infer that the good tasting cookies had a particular shape that was different from the shape of the bad tasting cookies. In a subsequent evaluative priming task, the pictures of both types of cookies were used as primes. Results revealed that participants liked the shape of the good tasting cookies better than the shape of the bad tasting cookies even though the shape and taste of the cookies never co-occurred. The results of both studies are in line with the idea that implicit evaluation can be based on the inferred proposition that a stimulus (a word, a shape) is related to something good or bad, even when this relation was never experienced directly.

Effects of a single instruction or inference on implicit evaluation challenge association-activation models in which the formation of associations requires overlearning and thus multiple direct pairings. One could of course explain those results on the basis of association-activation models in which even a single instruction or inference suffices to create strong associations through which activation can spread. Regardless of whether such post-hoc models are plausible, the most important point in the context of the present paper is that empirical research on instruction- or inference-based implicit evaluation was inspired by the novel idea that propositions might mediate implicit evaluations. Hence, this research illustrates the predictive value of propositional models of implicit evaluation.

\section{Implicit Evaluation is Moderated by Relational Information}

Unlike simple associations, propositions contain information about how concepts are related. Hence, if implicit evaluation is mediated by propositional information, relational information could moderate implicit evaluation. Peters and Gawronski (2011; see Zanon, De 
Houwer, \& Gast, 2012, and Zanon, De Houwer, Gast, \& Smith, in press, for conceptual replications) tested this hypothesis by pairing pictures of two unknown persons (person A and person B) most often with positive trait words and pictures of two other unknown persons (person C and person D) most often with negative trait words. Importantly, they also manipulated the information that participants received about how the unknown persons were related to the trait words. More specifically, persons A and C were said to possess the traits described by the trait words with which they were paired whereas persons B and D were said to possess the traits opposite to those implied by the trait words with which they were paired. Implicit evaluations (as captured by effects in the evaluative priming task or the affect misattribution procedure) were more positive for A than for C, which is in line with the fact that A was paired with positive traits and $\mathrm{C}$ was paired with negative traits. The implicit evaluation of $\mathrm{B}$, however, was more negative than that of $\mathrm{D}$, despite the fact that $\mathrm{B}$ was actually paired with positive traits whereas D was seen together with negative traits. Hence, the manipulation of relational information (e.g., person A has that trait versus person B has the opposite trait) affected the implicit evaluation of the unknown persons. Interestingly, relational information had a bigger impact when it was presented during the person-trait pairings then when it was presented only after the pairings.

From the perspective of propositional models, these findings indicate that implicit evaluations are determined not by actual events (e.g., the co-occurrence of B and positive traits) but by the propositions that are formed on the basis of those events (e.g., that B has negative traits). The fact that relational information has less impact when it is available only after the relevant stimuli have been presented, can be explained if it is assumed that the pairing of stimuli by default (i.e., without evidence to the contrary) leads to the proposition that the stimuli are somehow equivalent (e.g., that person A has the trait it is paired with). In 
line with this assumption, Zanon et al. (in press) observed that explicit relational information about the equivalence of paired stimuli (i.e., instructing participants that stimuli are similar in meaning) added nothing to the effect of merely pairing the stimuli. In contrast, explicit relational information that implied a relation of opposition (i.e., instructing participants that stimuli have an opposite meaning) counteracted the effect of stimulus pairings on implicit evaluations.

Again one could conceive of post-hoc ways in which association-activation models could account for these data. For instance, one could argue that participants recode information on the basis of the available relational information (e.g., pairing person B with the trait HAPPY in the context of the relational cue "opposite" would lead to a recoding of HAPPY into SAD) and that associations are formed on the basis of the recoded information (e.g., B would be associated with SAD; see Peters \& Gawronski, 2011). Although one could question the viability of such a post-hoc account, the main point I want to make is that studies on the impact of relational information were conceived of because of the idea that propositions might mediate implicit evaluations, thus demonstrating that this idea can lead to the discovery of important new facts about implicit evaluation.

\section{Implicit Evaluation Can Reflect Different Propositions}

Propositional models of implicit evaluation postulate that instances of implicit evaluation are based on propositional knowledge. For instance, if a prime stimulus such as the word "ME” facilitates responding to a positive target in an evaluative priming task, it does so not because the concept node "self" is associated in memory with the concept node "good” but because participants automatically form or activate a proposition about the relation between the concepts "I" and "good”. As indicated earlier on in this paper, the same two concepts can be related in different ways. In some cases, different propositions might 
lead to the same implicit evaluation, that is, to the same effect of stimuli on evaluative responses. For instance, the prime "ME” might facilitate responding to a positive target either because of the proposition "I am good" or because of the proposition "I want to be good".

This novel perspective sheds a new light on a number of puzzling findings in the literature on implicit evaluation. For instance, it has repeatedly been observed that the implicit evaluation of self-related stimuli (as indexed by a variety of tasks such as the IAT, name-letter task, and extrinsic affective Simon task) is as positive in depressed patients as in non-depressed controls (e.g., De Raedt, Schacht, Franck, \& De Houwer, 2006). In fact, depressed patients with suicidal thoughts even reveal a more positive implicit evaluation of the self than matched controls (Franck, De Raedt, Dereu, \& Van den Abbeele, 2007). One possible explanation of these findings is that the implicit evaluation of self-related stimuli in depressed patients reflects the proposition "I want to be good" but reflects the proposition "I am good” in non-depressed controls. Whereas the second proposition underlies actual selfesteem, the first proposition might provide the foundation of ideal self-esteem (see Zentner \& Renaud, 2007, for a discussion of the distinction between these two types of self-esteem). Whereas actual self-esteem is probably lower in depressed than in non-depressed individuals, there are many indications that ideal self-esteem is more extremely positive in depressed than in non-depressed individuals.

To test this idea, Remue, De Houwer, Barnes-Holmes, Vanderhasselt, and De Raedt (2013) asked students with a strong or a weak tendency for depression to complete two versions of the Implicit Relational Assessment Procedure (IRAP; see D. Barnes-Holmes, Y. Barnes-Holmes, Stewart, \& Boles, 2010). The IRAP was developed specifically to test how stimuli are related by asking participants to respond as if stimuli are related in a certain way. In the first IRAP that was used by Remue et al., on each trial the label "I am” or "I am not" 
appeared on the screen together with a positive or negative adjective. In some blocks, participants were required to respond as if they had a positive self-esteem. More specifically, they had to select the response "true" whenever the information on the screen implied positive self-esteem (e.g., "I am valuable” or "I am not rejected”) and to select the response "false” whenever the information on the screen implied negative self-esteem (e.g., "I am rejected” or “I am not valuable”). In other blocks, participants were asked to respond as if they had negative self-esteem (e.g., press true for "I am rejected” and “I am not valuable”; press false for “I am valuable” and “I am not rejected”). The difference in performance on both types of blocks was seen as an index of actual self-esteem. The second IRAP was identical to the first one except that the labels "I am" and "I am not" were replaced by the labels “I want to be” and “I don’t want to be”. On some blocks, participants responded as if they wanted to be good (e.g., press true for "I want to be valuable” and "I don't want to be rejected”; press false for “I want to be rejected” and “I don’t want to be valuable”) whereas on other blocks, they responded as if they wanted to be bad (e.g., press true for "I want to be rejected" and "I don’t want to be valuable" and press false for "I want to be valuable” and "I don't want to be rejected”). The difference in performance on those two blocks was seen as an index of ideal self-esteem. As predicted, participants with a strong tendency for depression scored lower than other participants on the index of actual self-esteem but higher on the index of ideal self-esteem.

In future studies, my colleagues and I aim to examine other puzzling findings in a similar manner. For instance, it was recently observed that hospitalized alcoholics (a) have an elevated automatic tendency to avoid alcohol stimuli and (b) are more likely to relapse when they have a strong automatic tendency to avoid alcohol stimuli (Spruyt et al., 2013). We plan to test the hypothesis that these results stems from a strong automatic endorsement of the 
proposition "I should avoid alcohol" rather than the proposition "I want to avoid alcohol".

In the context of the present paper, studies like these are important because they could provide additional evidence for propositional models of implicit evaluation. However, they could also tell us whether it is possible to measure all kinds of propositions (i.e., both evaluative and non-evaluative ones) indirectly using reaction time tasks. Indirect measures of propositional knowledge might provide a window into the spontaneous thoughts and beliefs of people. ${ }^{1}$ To the extent that (a) traditional questionnaires capture only well-considered opinions and beliefs and (b) spontaneous thoughts and beliefs can differ from well-considered opinions and beliefs, indirect measures of spontaneous thoughts and beliefs might provide an important new avenue for understanding and predicting behavior. Note that in certain variants of the IAT, some of the category labels include relational information such as "I like" and "I do not like” (Olson \& Fazio, 2004), “I want” and “I do not want” (Dewitte \& De Houwer, 2008), or “most people like” and “most people don’t like” (Yoshida, Peach, Zanna, \& Spencer, 2012). To the extent that the relational information in the labels actually influences the outcome of the IATs, one can conclude that the IAT effects provide an indirect measure of propositions rather than associations. It would indeed be difficult to explain the impact of relational information on IAT effects if those effects are mediated by representations that do not specify any relational information. Hence, these variants of the IAT can be described as propositionalized IATs.

\footnotetext{
${ }^{1}$ From a functional perspective, indirect measures of propositions can be conceptualized as instances of implicit relational responding, that is, as the impact of stimuli on relational responses (see Hughes, Barnes-Holmes, \& Vahey, 2012, for an excellent functional analysis of implicit cognition). Implicit evaluation can be seen as a subclass of implicit relational responding in that it involves one type of responses, namely evaluative responses. However, because the present paper focusses on the cognitive level of analysis (see De Houwer, 2011, and De Houwer et al., in press), we will not elaborate on these conceptual and meta-theoretical issues.
} 


\section{Conclusion}

Research on implicit evaluation has been dominated by association-activation theories from the start on (e.g., Fazio et al., 1986). I encourage researchers to consider an alternative view according to which implicit evaluation is mediated by the formation or activation of propositions. Doing so will lead to new questions and ideas about how and when instructions and inferences can result in implicit evaluations, whether or how those instruction- and inference-based implicit evaluations differ from experience-based implicit evaluations, the conditions under which relational information moderates implicit evaluations, and the different types of propositions that might underlie various instances of implicit evaluation. Although it will be difficult to disentangle association-activation and propositional models, research on implicit evaluation is bound to benefit from having a new kid on the block that provides a new perspective and new predictions. 


\section{References}

Aarts, H., \& Elliot, A. (2012). Goal-directed behavior. New York: Psychology Press.

Barnes-Holmes, D., Barnes-Holmes, Y, Stewart, I, \& Boles, S. (2010). A sketch of the Implicit Relational Assessment Procedure (IRAP) and the Relational Elaboration and Coherence (REC) model. The Psychological Record, 60, 527-542.

Collins, A. M., \& Loftus, E. F. (1975). A spreading activation of semantic processing. Psychological Review, 82, 407-428.

De Houwer, J. (2006). Using the implicit association test does not rule out an impact of conscious propositional knowledge on evaluative conditioning. Learning and Motivation, 37, 176-187.

De Houwer, J. (2009a). How do people evaluate objects? A brief review. Social and Personality Psychology Compass, 3, 36-48.

De Houwer, J. (2009b). The propositional approach to associative learning as an alternative for association formation models. Learning \& Behavior, 37, 1-20.

De Houwer, J. (2011). Why the cognitive approach in psychology would profit from a functional approach and vice versa. Perspectives on Psychological Science, 6, 202-209.

De Houwer, J. (in press). Why a propositional single-process model of associative learning deserves to be defended. In J. W. Sherman, B. Gawronski, \& Y. Trope (Eds.), Dual processes in social psychology. NY: Guilford.

De Houwer, J., Gawronski, B., \& Barnes-Holmes, D. (in press). A functionalcognitive framework for attitude research. European Review of Social Psychology.

Dehaene, S., Naccache, L., Le Clec’H, G., Koechlin, E., Mueller, M., DehaeneLambertz, G., et al. (1998). Imaging unconscious semantic priming. Nature, 395, 597-600.

De Raedt, R., Schacht, R., Franck, E., \& De Houwer, J. (2006). Self-esteem and 
depression revisited: Implicit positive self-esteem in depressed patients? Behaviour Research and Therapy, 44, 1017-1028.

Dewitte, M., \& De Houwer, J. (2008). Proximity and distance motives in adult attachment. European Journal of Personality, 22, 675-694.

Eagly, A. H., \& Chaiken, S. (2007). The advantages of an inclusive definition of attitude. Social Cognition, 25, 582-602.

Fazio, R. H. (2001). On the automatic activation of associated evaluations: An overview. Cognition and Emotion, 15, 115-141.

Fazio, R. H. (2007). Attitudes as object-evaluation associations of varying strength. Social Cognition, 25, 603-637.

Fazio, R. H., Sanbonmatsu, D. M., Powell, M. C., \& Kardes, F. R. (1986). On the automatic activation of attitudes. Journal of Personality and Social Psychology, 50, 229-238.

Franck, E., De Raedt, R., Dereu, M., \& Van den Abbeele, D. (2007). Implicit and explicit self-esteem in currently depressed individuals with and without suicidal ideation. Journal of Behavior Therapy and Experimental Psychiatry, 38, 75-85.

Garcia-Marques, L., \& Ferreira, M. B., (2011). Friends and foes of theory construction in psychological science: Vague dichotomies, unified theories of cognition, and the new experimentalism. Perspectives on Psychological Science, 6, 192-201.

Gast, A. \& De Houwer, J. (2012). Evaluative conditioning without directly experienced pairings of the conditioned and the unconditioned stimuli. The Quarterly Journal of Experimental Psychology, 65, 1657-1674.

Gast, A., \& De Houwer, J. (2013). The influence of extinction and counterconditioning instructions on evaluative conditioning effects. Learning and Motivation, 44, 312-325. 
Gawronski, B., \& Bodenhausen, G. V. (2011). The associative-propositional evaluation model: Theory, evidence, and open questions. Advances in Experimental Social Psychology, 44, 59-127.

Gawronski, B., \& Bodenhausen, G. V. (in press). Theory evaluation. In B. Gawronski, \& G. V. Bodenhausen (Eds.). Theory and explanation in social psychology. New York: Guilford Press.

Gregg, A. P., Banaji, M. R., \& Seibt, B. (2006). Easier made than undone: The asymmetric malleability of automatic preferences. Journal of Personality and Social Psychology, 90, 1-20.

Heider, M. N., Spruyt, A., \& De Houwer, J. (2014). Automatic task misapplication. Manuscript in preparation.

Hempel, C. G. (1970). Aspects of scientific explanation and other essays in the philosophy of science. New York: Free Press.

Herring, D. R., White, K. R., Jabeen, L. N., Hinojos, M., Terrazas, G., Reyes, S. M., Taylor, J. H., \& Crites, S. L., Jr. (2013). On the automatic activation of attitudes: A quarter century of evaluative priming research. Psychological Bulletin, 139, 1062-1089.

Hintzman, D. L. (1986). “Schema abstraction” in a multipletrace memory model. Psychologicul Review, 93, 411-428.

Hughes, S., Barnes-Holmes, D., \& De Houwer, J. (2011). The dominance of associative theorising in implicit attitude research: Propositional and behavioral alternatives. The Psychological Record, 61, 465-498.

Hughes, S., Barnes-Holmes, D., \& Vahey, N. (2012). Holding on to our functional roots when exploring new intellectual islands: A voyage through implicit cognition. Journal of Contextual Behavioural Science, 1, 17-38. 
Klauer, K.C., Musch, J., \& Eder, A. B. (2005). Priming of semantic classifications: Late and response-related, or earlier and more central? Psychonomic Bulletin and Review, 12, 897-903.

Klauer, K. C., Teige-Mocigemba, S., \& Spruyt, A. (2009). Contrast effects in spontaneous evaluations: A psychophysical account. Journal of Personality and Social Psychology, 96, 265-287.

Lagnado, D. A., Waldmann, M. R., Hagmayer, Y., \& Sloman, S. A. (2007). Beyond covariation: Cues to causal structure. In A. Gopnik \& L. Schulz (Eds.), Causal learning: Psychology, philosophy, and computation (pp. 154-172). Oxford: Oxford University Press.

Markus, H. R. (1977). Self-schemata and processing information about the self. Journal of Personality and Social Psychology, 35, 63-78.

Miller, R.R., \& Escobar, M. (2001). Contrasting acquisition-focused and performance-focused models of behavior change. Current Directions in Psychological Science, 10, 141-145.

Mitchell, C. J., De Houwer, J., \& Lovibond, P. F. (2009). The propositional nature of human associative learning. Behavioral and Brain Sciences, 32, 183-198.

Olson, M. A., \& Fazio, R. H. (2004). Reducing the influence of extra-personal associations on the Implicit Association Test: Personalizing the IAT. Journal of Personality and Social Psychology, 86, 653-667.

Peters, K. R., \& Gawronski, B. (2011). Are we puppets on a string? Comparing the impact of contingency and validity on implicit and explicit evaluations. Personality and Social Psychology Bulletin, 37, 557-569.

Remue, J., De Houwer, J., Barnes-Holmes, D., Vanderhasselt, M.-A., \& De Raedt, R. (2013). Self-esteem revisited: Performance on the implicit relational assessment procedure as 
a measure of self- versus ideal self-related cognitions in dysphoria. Cognition \& Emotion, 27, 1441-1449.

Rydell, R. J., \& McConnell, A. R. (2006). Understanding implicit and explicit attitude change: A systems of reasoning analysis. Journal of Personality and Social Psychology, 91, 995-1008.

Smith, C. T., De Houwer, J., \& Nosek, B. A. (2013). Consider the source: Persuasion of implicit evaluations is moderated by source credibility. Personality and Social Psychology Bulletin, 39, 193-205.

Strack, F., \& Deutsch, R. (2004). Reflective and impulsive determinants of social behavior. Personality and Social Psychology Review, 8, 220-247.

Spruyt, A., De Houwer, J., Tibboel, H., Verschuere, B., Crombez, G., Verbanck, P., Hanak, C., Brevers, D., \& Noël, X. (2013). On the predictive validity of automatically activated approach/avoidance tendencies in abstaining alcohol-dependent patients. Drug and Alcohol Dependence, 127, 81-86.

Van Opstal, F., Gevers, W., Osman, M., \& Verguts, T. (2010). Unconscious task application. Consciousness and Cognition, 19, 999-1006.

Yoshida, E., Peach, J.M., Zanna, M.P. \& Spencer, S.J. (2012). Not all automatic associations are created equal: How implicit normative evaluations are distinct from implicit attitudes and uniquely predict meaningful behavior. Journal of Experimental Social Psychology, 48, 694-706.

Zanon, R., De Houwer, J., \& Gast, A. (2012). Context effects in evaluative conditioning of implicit evaluations. Learning \& Motivation, 43, 155-165.

Zanon, R., De Houwer, J., \& Gast, A., \& Smith, C. (in press). When does relational information influence evaluative conditioning? Quarterly Journal of Experimental 
Psychology.

Zentner, M., \& Renaud, O. (2007). Origins of adolescents' ideal self: An intergenerational perspective. Journal of Personality and Social Psychology, 92, 557-574. 


\section{List of Figures}

Figure 1. A schematic representation of implicit evaluation (A), an associationactivation model of implicit evaluation (B), and a propositional model of implicit evaluation (C). Elements in the squares represent mental processes and representations that mediate the impact of stimuli in the environment on evaluative responses. 
(A)

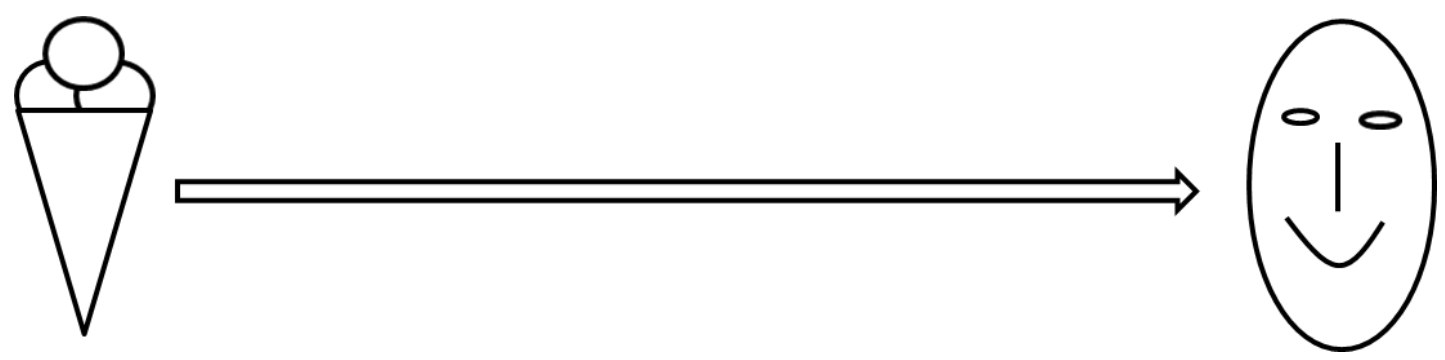

(B)

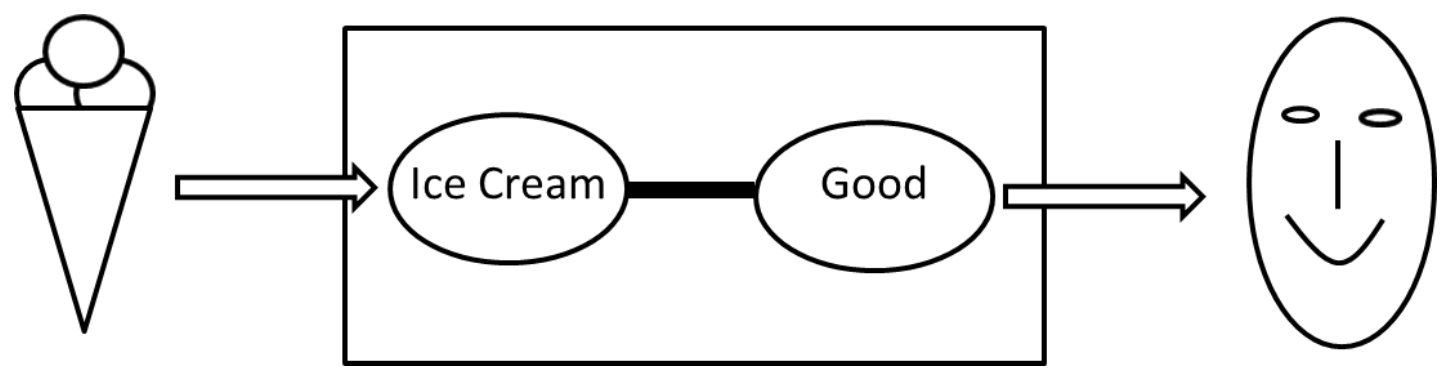

(C)

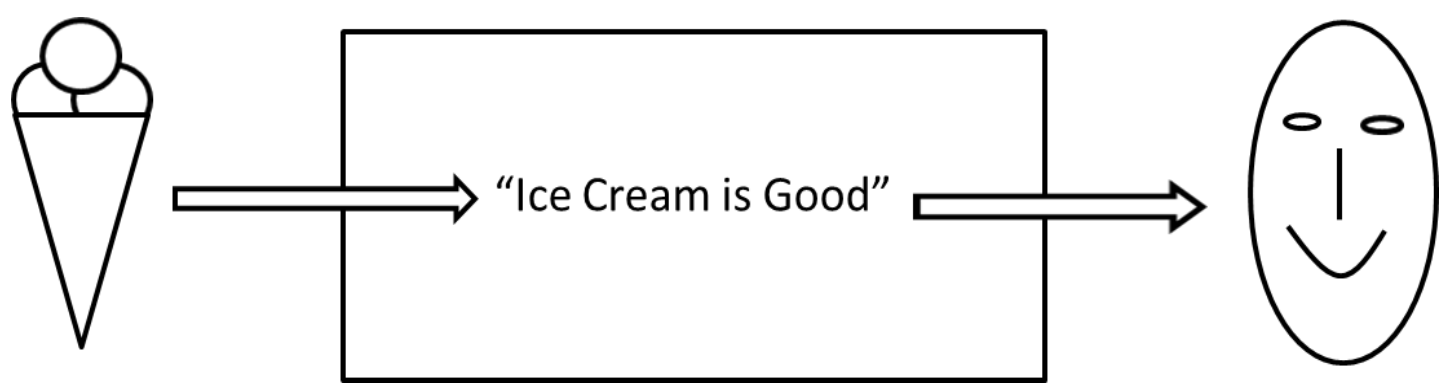

